\title{
Stereoselective Oxidation Kinetics of Deoxycholate in Recombinant and Microsomal CYP3A Enzymes: Deoxycholate 19-Hydroxylation Is an In Vitro Marker of CYP3A7 Activity ${ }^{\mathbb{S}}$
}

\author{
Yu-Jie Chen, ${ }^{1}$ Jian Zhang, ${ }^{1}$ Ping-Ping Zhu, Xian-Wen Tan, Qiu-Hong Lin, Wen-Xia Wang, \\ Shan-Shan Yin, Ling-Zhi Gao, Ming-Ming Su, Chang-Xiao Liu, Liang Xu, Wei Jia, \\ Irina F. Sevrioukova, and $\mathbb{O}$ Ke Lan
}

Key Laboratory of Drug-Targeting and Drug Delivery System of the Education Ministry, West China School of Pharmacy, Sichuan University, Chengdu, People's Republic of China (Y.-J.C., J.Z., P.-P.Z., X.-W.T., Q.-H.L., W.W., S.-S.Y., L.-Z.G., L.X., K.L.); Metabolomics Shared Resource, University of Hawaii Cancer Center, Honolulu, Hawaii (M.-M.S., W.J.); Department of Molecular Biology and Biochemistry, University of California, Irvine, California (I.F.S.); State Key Laboratory of Drug Delivery Technology and Pharmacokinetics, Tianjin Institute of Pharmaceutical Research, Tianjin, People's Republic of China (C.-X.L.); and Chengdu Health-Balance Medical Technology Co., Ltd., Chengdu, People's Republic of China (S.-S.Y., K.L.)

Received February 5, 2019; accepted March 25, 2019

\section{ABSTRACT}

The primary bile acids (BAs) synthesized from cholesterol in the liver are converted to secondary BAs by gut microbiota. It was recently disclosed that the major secondary $B A$, deoxycholate (DCA) species, is stereoselectively oxidized to tertiary BAs exclusively by CYP3A enzymes. This work subsequently investigated the in vitro oxidation kinetics of DCA at $\mathrm{C}-1 \beta, \mathrm{C}-3 \beta, \mathrm{C}-4 \beta$, $\mathrm{C}-5 \beta, \mathrm{C}-6 \alpha, \mathrm{C}-6 \beta$, and $\mathrm{C}-19$ in recombinant CYP3A enzymes and naive enzymes in human liver microsomes (HLMs). The stereoselective oxidation of DCA fit well with Hill kinetics at 1-300 $\mu \mathrm{M}$ in both recombinant CYP3A enzymes and pooled HLMs. With no contributions or trace contributions from CYP3A5, CYP3A7 favors oxidation at $\mathrm{C}-19, \mathrm{C}-4 \beta, \mathrm{C}-6 \alpha, \mathrm{C}-3 \beta$, and $\mathrm{C}-1 \beta$, whereas CYP3A4 favors the oxidation at $C-5 \beta$ and $C-6 \beta$ compared with each other. Correlation between DCA oxidation and testosterone
$6 \beta$-hydroxylation in 14 adult single-donor HLMs provided proofof-concept evidence that DCA 19-hydroxylation is an in vitro marker reaction for CYP3A7 activity, whereas oxidation at other sites represents mixed indicators for CYP3A4 and CYP3A7 activities. Deactivation caused by DCA-induced cytochrome P450-cytochrome P420 conversion, as shown by the spectral titrations of isolated CYP3A proteins, was observed when DCA levels were near or higher than the critical micelle concentration (about $1500 \mu \mathrm{M}$ ). Unlike CYP3A4, CYP3A7 showed abnormally elevated activities at 500 and $750 \mu \mathrm{M}$, which might be associated with an altered affinity for DCA multimers. The disclosed kinetic and functional roles of CYP3A isoforms in disposing of the gut bacteria-derived DCA may help in understanding the structural and functional mechanisms of CYP3A.

\section{Introduction}

Human CYP3A enzymes have important functions in the metabolism of drugs, toxicants (de Montellano, 2009), and endogenous steroids (Niwa et al., 2015). The CYP3A locus is located on chromosome 7q21.1 and consists of the following four genes: CYP3A4, CYP3A5, CYP3A7,

This work was supported in part by the National Institutes of Health Grant ES025767 (I.F.S.). The content is solely the responsibility of the authors and does not necessarily represent the official views of the National Institutes of Health.

${ }^{1}$ Y.-J.C. and J.Z. contributed equally to this work.

https://doi.org/10.1124/dmd.119.086637.

$\mathbf{S}$ This article has supplemental material available at dmd.aspetjournals.org. and CYP3A43. CYP3A43 is the most recently identified member, which is expressed in prostate and testis, and whose functional role is not yet understood (Gellner et al., 2001). CYP3A4 is the major drug-metabolizing cytochrome P450 (P450) isoenzyme predominantly expressed in the adult human liver and small intestine (Shimada et al., 1994; Paine et al., 2006; Michaels and Wang, 2014). Compared with CYP3A4, CYP3A5 expresses at lower levels in adults (Koch et al., 2002; Drozdzik et al., 2018) and has a high similarity in the substrate specificity (Williams et al., 2002; Patki et al., 2003). There is high intraindividual and interindividual variability in the expression and activity of CYP3A4 and CYP3A5, which together are estimated to metabolize at least $50 \%$ of the currently prescribed drugs (Wienkers and Heath, 2005; Rendic and

ABBREVIATIONS: BA, bile acid; CA, cholic acid $\left(3 \alpha, 7 \alpha, 12 \alpha\right.$-trihydroxy-5 $\beta$-cholan-24-oic acid); $\mathrm{CL}_{\text {int }}$, intrinsic clearance; $\mathrm{CMC}$, critical micelle concentration; DCA, deoxycholic acid ( $3 \alpha, 12 \alpha$-dihydroxy-5 $\beta$-cholan-24-oic acid); DCA- $1 \beta$-ol, $3 \alpha, 1 \beta, 12 \alpha$-trihydroxy-5 $\beta$-cholan-24-oic acid;

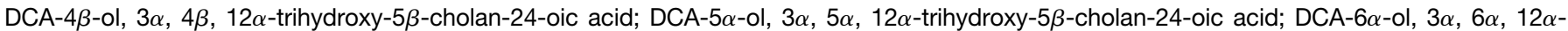
trihydroxy-5 $\beta$-cholan-24-oic acid; DCA-6 $\beta$-ol, $3 \alpha, 6 \beta, 12 \alpha$-trihydroxy-5 $\beta$-cholan-24-oic acid; DCA-19-ol, $3 \alpha, 12 \alpha, 19$-trihydroxy-5 $\beta$-cholan-24-oic acid; 3-dehydroDCA, 3-oxo, $12 \alpha$-hydroxy-5 $\beta$-cholan-24-oic acid; DHEA, dehydroepiandrosterone; GDCA, glycodeoxycholate; HLM, human liver microsome; LC, liquid chromatography; MS, mass spectrometry; $\mathrm{m} / \mathrm{z}$, mass/charge ratio; NADPH-A, NADPH regenerating system solution A; NADPH-B, NADPH regenerating system solution $B ; P 450$, cytochrome P450; $S_{50}$, substrate concentration occupying half of the binding sites; TDCA, taurodeoxycholate. 
Guengerich, 2015). On the contrary, CYP3A7 is expressed specifically in the fetal and neonatal liver (Schuetz et al., 1993), and plays a critical role in placental estriol synthesis by controlling $16 \alpha$ hydroxylation of dehydroepiandrosterone (DHEA) sulfate (Kitada et al., 1987a,b). There is a progressive change in the postnatal expression from CYP3A7 toward CYP3A4, leading to a generally negligible expression of CYP3A7 in adult livers (Lacroix et al., 1997; Stevens et al., 2003). The developmental transition of CYP3A7 to CYP3A4 during childhood is of particular importance in pediatric pharmacology (Stevens, 2006).

Despite the high degree of sequence identity $(>81.9 \%)$ (Gellner et al., 2001), CYP3A4, CYP3A5, and CYP3A7 demonstrate subtle differences in the substrate specificity, catalytic efficiency, and/or metabolic regioselectivity (Williams et al., 2002). A number of exogenous drug molecules and endogenous steroids, including midazolam, alprazolam, triazolam, alfentanil, erythromycin, vincristine, quinidine, testosterone, and cortisol, have been proposed as probe substrates for CYP3A4/5 activities (Liu et al., 2007). Currently, midazolam 1-hydroxylation and testosterone $6 \beta$-hydroxylation are the mostly used in vitro marker reactions of CYP3A4/5. To our knowledge, the only two CYP3A7 marker reactions mentioned in the literature are DHEA $16 \alpha$-hydroxylation and testosterone $2 \alpha$-hydroxylation. One group showed that the intrinsic clearance $\left(C l_{\text {int }}\right)$ of recombinant CYP3A7 for DHEA $16 \alpha$-hydroxylation is three times higher than that of CYP3A4 (Stevens et al., 2003). According to another study (Leeder et al., 2005), the difference in metabolite formation is only 1.3-fold and 2.6-fold for DHEA 16 $\alpha$-hydroxylation and testosterone $2 \alpha$-hydroxylation, respectively. Also, a ratio between the $2 \alpha / 6 \beta$ hydroxylated metabolites produced by CYP3A7 depends on the substrate levels, and, thus, testosterone $2 \alpha$-hydroxylation cannot serve as a biomarker for CYP3A7 activity (Kandel et al., 2017). Without the isoform-specific probe reactions, it is highly challenging to investigate structure-function relations and relative metabolic activities of CYP3A enzymes, particularly for the most poorly studied CYP3A7.

Deoxycholic acid (DCA) is a secondary bile acid (BA) derived from 7-dehydroxylation of cholic acid (CA), the primary BA synthesized from cholesterol in hepatocytes (Russell, 2003). Gut bacteria carrying a BA-inducible gene, such as Clostridium and Eubacterium, are responsible for the multistep 7-dehydroxylation reactions of CA (Ridlon et al., 2006; Dawson and Karpen, 2015). It was recently disclosed that CYP3A4 and CYP3A7 are exclusively responsible for the tertiary regioselective oxidation of DCA at $\mathrm{C}-1 \beta, \mathrm{C}-3 \beta, \mathrm{C}-4 \beta, \mathrm{C}-5 \beta, \mathrm{C}-6 \alpha$, C-6 $\beta$, and C-19 (Fig. 1) (Zhang et al., 2019). The identification of the tertiary BA metabolic pathways allowed better understanding and expansion of the biologic functions of CYP3A4 and CYP3A7, which were suggested to have an inherent role in the stress response to the bacteria-produced secondary BAs. Here, we report the oxidation kinetics of DCA in recombinant and microsomal CYP3A enzymes and the binding affinity of DCA for isolated CYP3A proteins. It was shown that DCA 19-hydroxylation at low substrate levels $(\leq 300 \mu \mathrm{M})$ is an in vitro marker reaction for CYP3A7 activity, whereas DCA oxidation at other sites can be used as mixed in vitro indicators for CYP3A4 and CYP3A7 activities.

\section{Materials and Methods}

Materials and Reagents. DCA, DCA sodium salt, and CA-2,2,4,4- $\mathrm{D}_{4}$ were purchased from Sigma-Aldrich (St. Louis, MO). 3-DehydroDCA was purchased from Toronto Research Chemicals (Toronto, ON, Canada). DCA- $1 \beta$-ol, DCA- $4 \beta$-ol, DCA- $5 \beta$-ol, DCA- $6 \beta$-ol, DCA- $6 \alpha$-ol, and DCA-19-ol were synthesized as described in our recent report (Zhang et al., 2019). NADPH regenerating system solution A (NADPH-A; containing $26 \mathrm{mM}$ $\mathrm{NADP}^{+}, 66 \mathrm{mM}$ glucose-6-phosphate, and $66 \mathrm{mM} \mathrm{MgCl}_{2}$ in $\mathrm{H}_{2} \mathrm{O}$ ), NADPH regenerating system solution B (NADPH-B; containing $40 \mathrm{U} / \mathrm{ml}$ glucose6-phosphate dehydrogenase in $5 \mathrm{mM}$ sodium citrate), and 0.5 M, pH 7.4 PBS were purchased from Corning (Tewksbury, MA). liquid chromatography (LC) with mass spectrometry (MS)-grade methanol, acetonitrile, and formic acid were purchased from Sigma-Aldrich. DMSO was purchased from Thermo Fisher Scientific (Waltham, MA). Ultrapure water was obtained by using a Milli-Q System (Millipore Sigma, Bedford, MA).

Human Liver Microsomes and Recombinant CYP3A Enzymes. The pooled human liver microsomes (HLMs) from 150 mixed-sex adult donors were purchased from Corning (Corning, NY). Fourteen single-donor HLMs were obtained from BD Biosciences (Woburn, MA). The donor information and testosterone $6 \beta$-hydroxylation activities were provided by the vendor and are summarized in Supplemental Table 1. The recombinant CYP3A4, CYP3A5, and CYP3A7 enzymes prepared from plasmid-transfected Escherichia coli (Bactosomes) were obtained from Cypex Ltd. (Dundee, UK). The in vitro experiments using human organ materials were reviewed and approved by the Institutional Review Board of the West China School of Pharmacy, Sichuan University.

In Vitro Activity Assay of DCA Oxidation. In vitro metabolism of DCA was assessed using the protocol described in our recent report (Zhang et al., 2019). In brief, stock solutions of DCA and ketoconazole were prepared in DMSO. Incubations with an initial substrate concentration of 1.0, 5.0, 25, 50, 100, 200, $300,500,750,1000,3000$, and $5000 \mu \mathrm{M}$ were performed in 96 -well plates in a shaking incubator at $37^{\circ} \mathrm{C}$. The $100-\mu$ lincubation solution contained $0.1 \mathrm{M}$ PBS (pH 7.4), $5.0 \mu \mathrm{l}$ of NADPH-A, $1.0 \mu \mathrm{l}$ of NADPH-B, $0.5 \mu \mathrm{l}$ of DCA working solution, $0.5 \mu \mathrm{l}$ of blank solvent or ketoconazole working solution, and $2.5 \mu \mathrm{l}$ of HLMs (protein concentration, $20 \mathrm{mg} / \mathrm{ml}$ ) or recombinant CYP3A enzymes (1.0 nmol protein/ml). The final protein concentration in

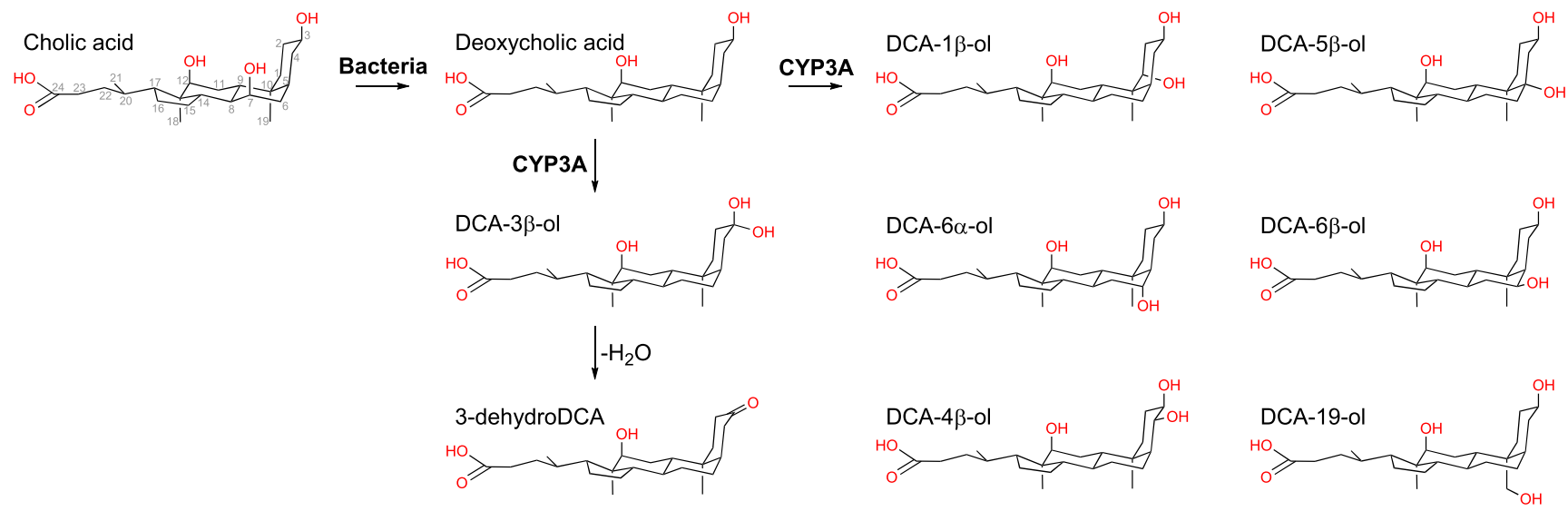

Fig. 1. CYP3A catalyzed tertiary oxidation of DCA in the host-gut microbial cometabolism of BAs. 
the incubation media was $0.5 \mathrm{mg} / \mathrm{ml}$ for HLMs and $50 \mathrm{pmol} / \mathrm{ml}$ for recombinant enzymes. The DMSO concentration in all incubations was $1 \%(\mathrm{v} / \mathrm{v})$. The inhibition of DCA oxidation $(50 \mu \mathrm{M})$ by ketoconazole $(0.001$, $0.01,0.03,0.1,0.5,1,5$, and $30 \mu \mathrm{M}$ ) was tested in the pooled HLMs. All incubations were performed in triplicate. The reactions were initiated after a 5 -minute preincubation period at $37^{\circ} \mathrm{C}$ by adding HLMs or recombinant enzymes and were stopped at a preset time point by adding $300 \mu \mathrm{l}$ of ice-cold acetonitrile containing $0.1 \%$ formic acid and $50 \mu \mathrm{M}$ CA-2,2,4,4-D4 as an internal standard. The reaction mixture was then centrifuged at $4{ }^{\circ} \mathrm{C}$ at $4000 \mathrm{~g}$ for 20 minutes. The supernatant $(50 \mu \mathrm{l})$ was diluted with $50 \mu \mathrm{l}$ water and subjected to LC-tandem MS (LC-MS/MS) analysis.

Quantitative LC-MS/MS Analysis. Quantitative analysis of DCA metabolites was performed on ACQUITY ultra-performance liquid chromatography coupled to a Xevo TQ-S mass spectrometer (Waters, Milford, MA) (Yin et al., 2017; Zhu et al., 2018). The mobile phases consisted of $0.01 \%$ formic acid in water (mobile phase A) and acetonitrile (mobile phase B). Five microliters of each sample was injected onto an ACQUITY BEH C18 Column $(1.7 \mu \mathrm{m}$, $100 \times 2.1 \mathrm{~mm})($ Waters $)$. The flow rate was $0.45 \mathrm{ml} / \mathrm{min}$ with the following mobile phase gradient: $0.0-0.5$ minute $(95 \% \mathrm{~A}), 0.5-1.0$ minute $(95 \%-64 \%$ A), 1.0-2.0 minutes $(64 \%-74 \%$ A), 2.0-4.0 minutes $(74 \%-70 \%$ A), 4.0-6.0 minutes (70\% A), 6.0-7.0 minutes (70\%-62\% A), 7.0-9.0 minutes $(62 \%-55 \%$ A), 9.0-12.5 minutes $(55 \%-30 \%$ A), 12.5-13.0 minutes (30\%-0\% A), 13.0-14.0 minutes (0\% A), 14.0-14.1 minutes (0\%-95\% A), and 14.1-15.0 minutes (95\% A). The mass spectrometer was operated in the negative mode with a $3.0-\mathrm{kV}$ capillary voltage. Selected ion recording at the mass transients of $\mathrm{m} / \mathrm{z} 389>389, \mathrm{~m} / \mathrm{z} 391>391$, and $\mathrm{m} / \mathrm{z} 407>407$ were used to detect 3-dehydroDCA, DCA, and hydroxylated DCA metabolites (DCA- $1 \beta$-ol, DCA- $4 \beta$-ol, DCA- $5 \beta$-ol, DCA- $6 \beta$-ol, DCA- $6 \alpha$-ol, and DCA19-ol), respectively. The source and desolvation temperatures were set at $150^{\circ} \mathrm{C}$ and $550^{\circ} \mathrm{C}$, respectively. The collision energy was set at $27 \mathrm{~V}$. Nitrogen and argon were used as cone and collision gases, respectively. The cone gas flow and desolvation gas flow were set at 150 and $950 \mathrm{l} / \mathrm{h}$, respectively. The metabolite concentrations were calculated by UNIFI (version 1.8; Waters). The metabolite formations as a function of substrate concentrations were fit to the Hill (sigmoidal) equation and hyperbolic (Michaelis-Menten) equation using GraphPad Prism software (version 7.0; GraphPad Software, La Jolla, CA).

Equilibrium Binding of DCA to the Full-Length CYP3A Enzymes. Codon-optimized genes for the full-length CYP3A5 and CYP3A7 with the C-terminal 4-histidine tag were produced by GENEWIZ (South Plainfield, NJ) and Synbio Technologies (Monmouth Junction, NJ), respectively, and cloned into pcWori expression vector. CYP3A7 was coexpressed with GroESL in E. coli $\mathrm{C} 41$ cells and purified using Ni-affinity and ion exchange chromatography, as previously described for CYP3A4 (Sevrioukova, 2017). CYP3A5 was coexpressed with GroESL in E. coli DH5 $\alpha$ cells and purified using the procedure developed for the truncated protein (Hsu et al., 2018). Equilibrium titrations were conducted at ambient temperature or $37^{\circ} \mathrm{C}$ in a Cary 300 spectrophotometer by adding small amounts of DCA (dissolved in DMSO) to the recombinant CYP3A enzymes $(2.5 \mu \mathrm{M})$ in $0.1 \mathrm{M}$ phosphate buffer ( $\mathrm{pH}$ 7.4) supplemented with $1 \mathrm{mM}$ dithiothreitol and $20 \%$ glycerol. The absolute or difference absorbance spectra were recorded 10-15 minutes after the addition of DCA aliquots. In the latter case, the equal volume of DMSO was added to the reference cuvette to correct for the solvent-induced spectral changes. In all experiments, the final DMSO concentration was $<5 \%$. The Hill coefficient and $\mathrm{S}_{50}$ values were estimated from sigmoidal fits to the plots of the maximal absorbance change versus DCA concentration using SigmaPlot (version 11.0; Systat Software, Inc., San Jose, CA).

Spectrophotometric Analysis of DCA Micelle Formation. Noninvasive spectrophotometric analysis was performed to determine the micelle formation of DCA in buffers used in the activity and binding assays (Reis et al., 2004). DCA stock solutions were prepared by overnight shaking at 10 and $5 \mathrm{mM}$ in 0.1 M PBS (pH 7.4) with and without $20 \%$ (v/v) glycerol, respectively. Serial dilutions of the stock with the corresponding media were performed in volumetric flasks. The testing concentrations were 50-5000 $\mu \mathrm{M}$ for glycerolfree media and 200-10,000 $\mu \mathrm{M}$ for media containing $20 \%$ glycerol. Measurements were made in quartz cells with a $1-\mathrm{cm}$ path at $22^{\circ} \mathrm{C}$. Spectra were recorded at $190-300 \mathrm{~nm}$ on a UV-2600 Spectrophotometer
(SHIMADZU, Kyoto, Japan) with the medium scan rate and a scan step of $0.2 \mathrm{~nm}$. The maximum absorbance wavelength $\left(\lambda_{\max }\right)$ and value $\left(\mathrm{A}_{\max }\right)$ were recorded and plotted as a function of DCA concentrations by using GraphPad Prism software (version 7.0; GraphPad Software). Critical micelle concentration (CMC) was calculated at the intersecting point of the linear regression lines according to the Lambert-Beer law.

\section{Results}

DCA Oxidation Kinetics in Recombinant CYP3A Enzymes. Supplemental Fig. 1 illustrates the time-dependent metabolite formation after DCA $(50 \mu \mathrm{M})$ was incubated in the recombinant CYP3A4, CYP3A5, and CYP3A7 enzymes (50 pmol protein/ml) for 720 minutes. Compared with CYP3A4 and CYP3A7, CYP3A5 had only limited activity for $3 \beta$-oxidation and trace activities for $1 \beta$ - and $5 \beta$-oxidation. $\mathrm{C}-1 \beta$ and $\mathrm{C}-3 \beta$ were the major oxidation sites of both CYP3A4 and CYP3A7, whereas C-19 and C-4 $\beta$ were the selective oxidation sites of CYP3A7. Most metabolites continued to increase for 720 minutes, except for 3-dehydroDCA, which is derived from dehydration of the $3 \beta$-hydroxylated metabolite. The formation of 3 -dehydroDCA was linear in CYP3A5 incubation and reached a plateau after $\sim 180$ minutes in CYP3A7 incubation.

An incubation time of 60 minutes was used thereafter to investigate DCA oxidation kinetics at various substrate levels. As shown in Fig. 2, a gradual loss of oxidation activities at substrate levels higher than $300-750 \mu \mathrm{M}$ was due to protein denaturation caused by the powerful detergent effects of DCA, which was evident from the CMC of DCA $(\sim 1500 \mu \mathrm{M})$ measured in the buffer used in the activity assay (Fig. 3A). Within the substrate range of 1-300 $\mu \mathrm{M}$, however, the oxidation kinetic data fit well with both the Hill and hyperbolic equations. The Hill equation (Table 1) provided a better fit for most reactions than the hyperbolic equation (Supplemental Table 2). The Hill $R^{2}$ coefficients (goodness of fit) for most fittings were $>0.99$, except for the less productive reactions, such as CYP3A4-catalyzed oxidation at $\mathrm{C}-4 \beta$ (0.9649) and CYP3A7-catalyzed oxidation at C-6 $\beta$ (0.9746). The Hill coefficients were $>1$ for all of the CYP3A4catalyzed reactions and $<1$ for the reactions catalyzed by CYP3A 5 and CYP3A7, indicating a positive and no or negative cooperativity, respectively. The $\mathrm{S}_{50}$ values of CYP3A4-catalyzed reactions (98.8-144 $\mu \mathrm{M})$ were markedly lower than those of CYP3A7 $(165.9-529 \mu \mathrm{M})$. The total $C L_{\text {int }}$ value decreased in turn for CYP3A7 ( 97 ml/min per picomol), CYP3A4 (55 ml/min per picomole), and CYP3A5 (2 $\mathrm{mL} / \mathrm{min}$ per picomole). CYP3A5 showed no or only a trace activity for oxidation at $\mathrm{C}-1 \beta, \mathrm{C}-3 \beta$, and $\mathrm{C}-5 \beta$, confirming that DCA oxidation is selectively catalyzed by CYP3A4 and CYP3A7 rather than by CYP3A5. The relative activities of CYP3A4 to CYP3A7 $\left(C L_{\text {int }}\right.$ ratio) gradually increased for the oxidation at $\mathrm{C}-19, \mathrm{C}-4 \beta, \mathrm{C}-6 \alpha, \mathrm{C}-3 \beta, \mathrm{C}-1 \beta, \mathrm{C}-5 \beta$, and C-6 $\beta$. No DCA 19-hydroxylation activity was detected in the recombinant CYP3A4 and CYP3A5 enzymes, indicating that DCA 19-hydroxylation might be a potential marker reaction for CYP3A7 activity.

Binding Affinity of DCA with Isolated CYP3A Enzymes. To investigate the binding affinity of isolated CYP3A enzymes for DCA, we tried to perform equilibrium titrations of recombinant full-length CYP3A enzymes with DCA in the buffer and at the temperature used in the activity assay. In a glycerol-free solution, however, even small amounts of DCA caused a conversion of active P450 to the inactive P420 (Supplemental Fig. 2). Likewise, an increase of temperature from $23^{\circ} \mathrm{C}$ to $37^{\circ} \mathrm{C}$ did not affect the spin transition (Supplemental Fig. 3) but led to a decrease in protein stability, as all CYP3A isoforms precipitated at lower DCA concentrations $(\sim 1500 \mu \mathrm{M})$, which was 
3-dehydroDCA

DCA-1 $\beta$-ol
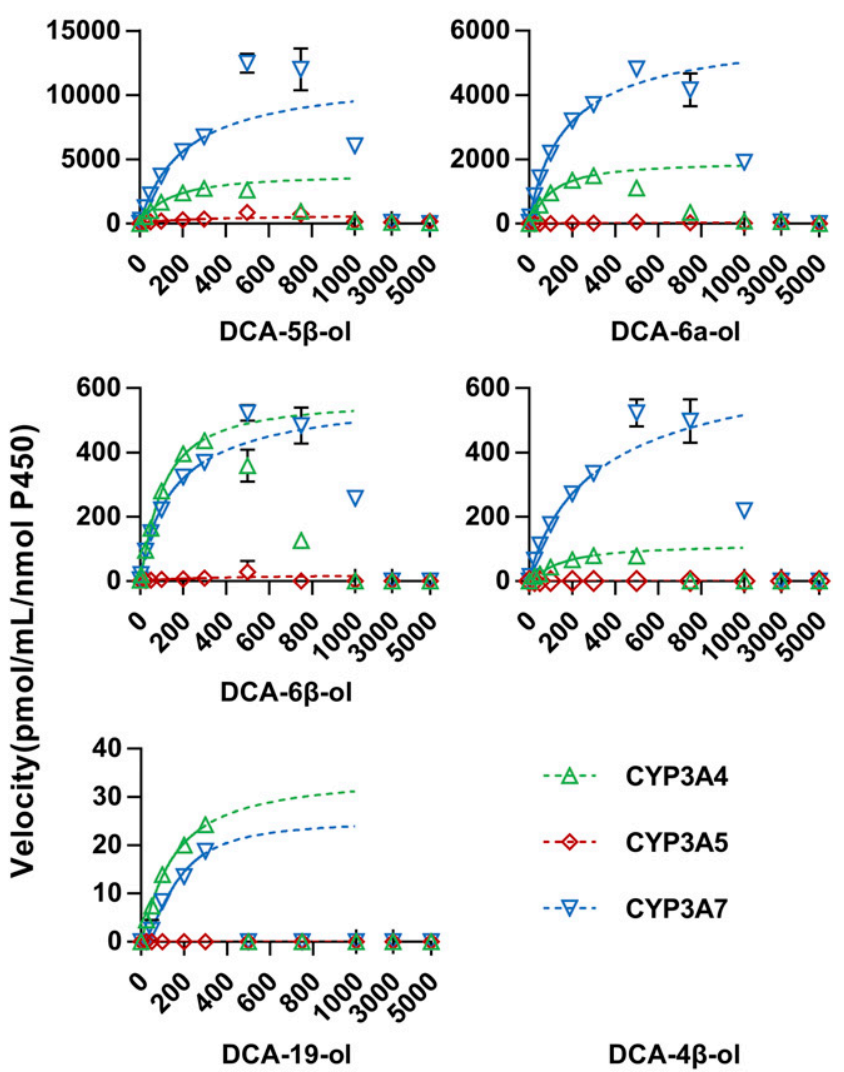

$-\diamond-\quad$ CYP3A5

$--\nabla-\cdot \quad$ CYP3A7

DCA-4ß-ol
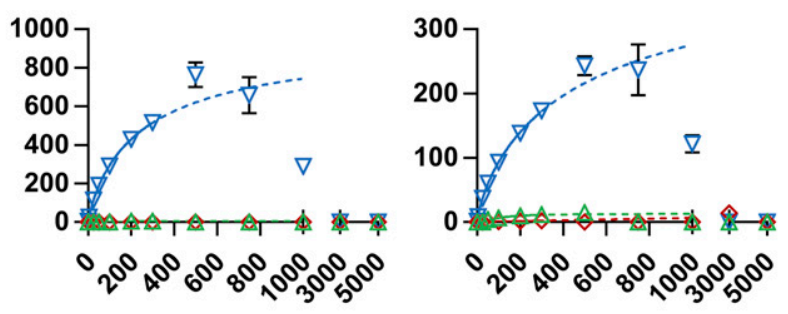

[DCA] $(\mu \mathrm{M})$

Fig. 2. Kinetics of DCA oxidation catalyzed by the recombinant CYP3A4, CYP3A5, and CYP3A7 enzymes (50 pmol protein/ml) fitted with the Hill equation $(1-300 \mu \mathrm{M})$. The dotted extrapolations of Hill kinetic curves demonstrate the violation of enzyme kinetics at higher substrate levels $(>300 \mu \mathrm{M})$. Each data point represents the average of three measurements, with error bars representing the S.D.

consistent with the CMC data measured in the corresponding buffer (Fig. 3B). The lengthy binding assays were therefore conducted at ambient temperature in the presence of $20 \%$ glycerol for protein stabilization. As shown in Fig. 4, DCA induced a high spin shift (type I spectral change) in all three isoforms, meaning that it can reach the heme iron and displace the distal water ligand. CYP3A4 could endure high DCA concentrations (up to $3600 \mu \mathrm{M}$ ) and underwent the largest high-spin shift $(\sim 47 \%)$. CYP3A5 and CYP3A7 had lower tolerability for DCA (up to $2000 \mu \mathrm{M}$ ), with a maximal high-spin conversion of only $\sim 30 \%$ and $33 \%$, respectively. Even at this DCA range, CYP3A5 was partially losing heme absorption (compare insets in Fig. 4, B and C), indicating the lowest stability of CYP3A5 in the presence of DCA and partially explaining its lowest catalytic ability for DCA oxidation. In accord with the activity assays, only CYP3A4 was found to display positive cooperativity in DCA binding (Table 2). This is evident from the sigmoidal shape of the

titration plot that can be fit with the Hill equation with $n=1.45$. In contrast, the respective plots for CYP3A5 and CYP3A7 (Fig. 4D) could be fit equally well with the hyperbolic and Hill equations with $n$ equal to $\sim 1$, indicating the lack of binding cooperativity. Further, a large difference in the $S_{50}$ values measured by the two methods suggests that the isolated soluble proteins have lower affinity for DCA than the respective isoforms incorporated in Bactosomes. This could be due to conformational changes and/or partitioning of DCA into the lipid bilayer and better access to the P450 active site.

DCA Oxidation Kinetics in Pooled HLMs and Inhibitory Effects of Ketoconazole. It was reported (Sim et al., 2005) that the fetusspecific CYP3A7 is also expressed in the livers of approximately $10 \%$ of adults. Consistently, the CYP3A7-specific activity of DCA 19-hydroxylation was also detected within the substrate range of $1-300 \mu \mathrm{M}$ in the pooled HLM of 150 mixed-sex adult donors (Supplemental Table 3). Because CYP3A4 prefers $5 \beta$-hydroxylation compared with CYP3A7, the metabolite ratios of DCA- $4 \beta$-ol/ DCA-5 $\beta$-ol and DCA-19-ol/DCA-5 $\beta$-ol were calculated to determine the variation of stereoselective oxidation at various substrate levels. As shown in Supplemental Fig. 4, the metabolite ratios for the recombinant CYP3A4 and CYP3A7 enzymes remained constant within the substrate range of $1-300 \mu \mathrm{M}$, but the respective values for HLMs decreased, with the substrate level increasing. Since both CYP3A4 and CYP3A7 proteins were present in the pooled HLM material, the decreased metabolite ratios in HLMs could be well explained by the lower $\mathrm{S}_{50}$ value for the CYP3A4-catalyzed $5 \beta$-hydroxylation $(102 \mu \mathrm{M})$ relative to that for the CYP3A7catalyzed 19-hydroxylation $(217 \mu \mathrm{M})$.

The inhibitory effects of ketoconazole on DCA oxidation were subsequently determined in the pooled HLMs (Supplemental Fig. 5). Ketoconazole had the highest $\mathrm{IC}_{50}$ value $(0.60 \mu \mathrm{M})$ for the CYP3A7specific DCA 19-hydroxylation. In agreement with the increasing contribution of CYP3A 4 to DCA oxidation at C- $4 \beta, \mathrm{C}-6 \alpha, \mathrm{C}-3 \beta$, $\mathrm{C}-1 \beta$, and $\mathrm{C}-5 \beta$, the respective $\mathrm{IC}_{50}$ values were reduced to 0.47 , $0.22,0.21,0.15$, and $0.13 \mu \mathrm{M}$. The most CYP3A4-favored DCA $6 \beta$ hydroxylation had the lowest $\mathrm{IC}_{50}$ value $(0.089 \mu \mathrm{M})$, which is consistent with the data measured with known marker reactions in HLMs (Patki et al., 2003). The data consistency indicated that the in vitro DCA oxidation reactions might be useful to discriminate CYP3A4 and CYP3A7 activities by using DCA 19-hydroxylation as a probe reaction for CYP3A7 activity.

Correlation of DCA Oxidation and Testosterone $6 \beta$-Hydroxylation in Individual HLMs. Metabolite formations of $50 \mu \mathrm{M}$ DCA were analyzed in a panel of HLMs from 14 single adult donors to further test the in vitro marker efficacy of DCA 19-hydroxylation for CYP3A7 activity. The CYP3A4-preferred testosterone $6 \beta$ hydroxylation was selected as a control because CYP3A5 contributes to testosterone $6 \beta$-hydroxylation much less than to midazolam 1-hydroxylation (Williams et al., 2002). As shown in Fig. 5A, no correlation was observed between DCA 19-hydroxylation activities and testosterone $6 \beta$-hydroxylation activities in the tested individual HLMs. In contrast, some interdependence was observed for the DCA metabolism at other sites (Fig. 5B), particularly for the CYP3A4-favored C-6 $\beta(r=0.9145)$ and C-5 $\beta(r=0.9043)$ oxidation. The latter correlation became much stronger when the $1 \beta-, 3 \beta-, 4 \beta-, 5 \beta-, 6 \alpha-$, and $6 \beta$-hydroxylation activities were corrected by subtracting the CYP3A7 contributions (Fig. 5C), which was achieved by calculating the CYP3A7 protein levels in single-donor HLMs and estimating the respective activities derived from CYP3A7 according to the activity data acquired in the recombinant CYP3A7 enzymes at $50 \mu \mathrm{M}$ DCA. Some of 
A
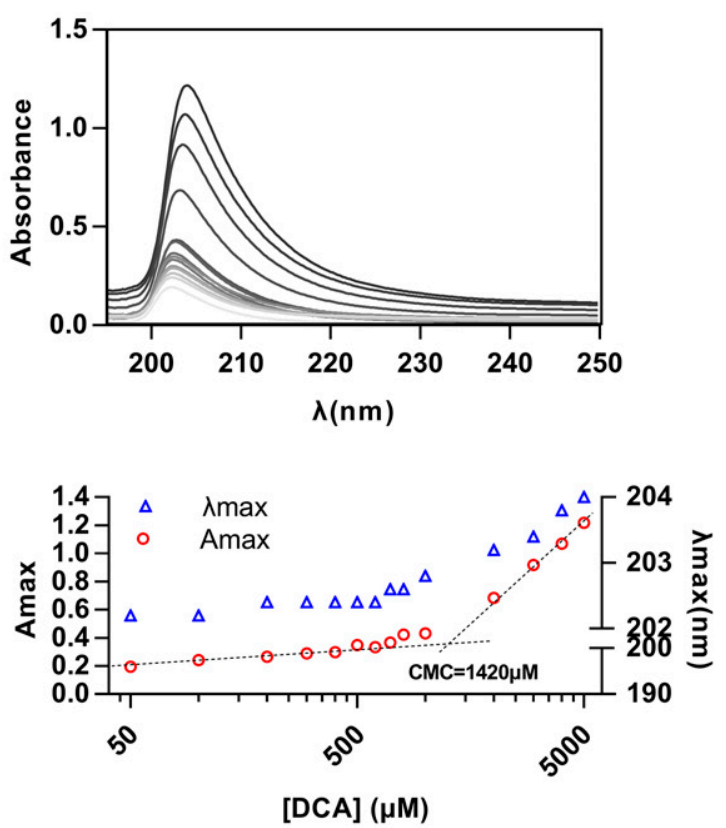

C

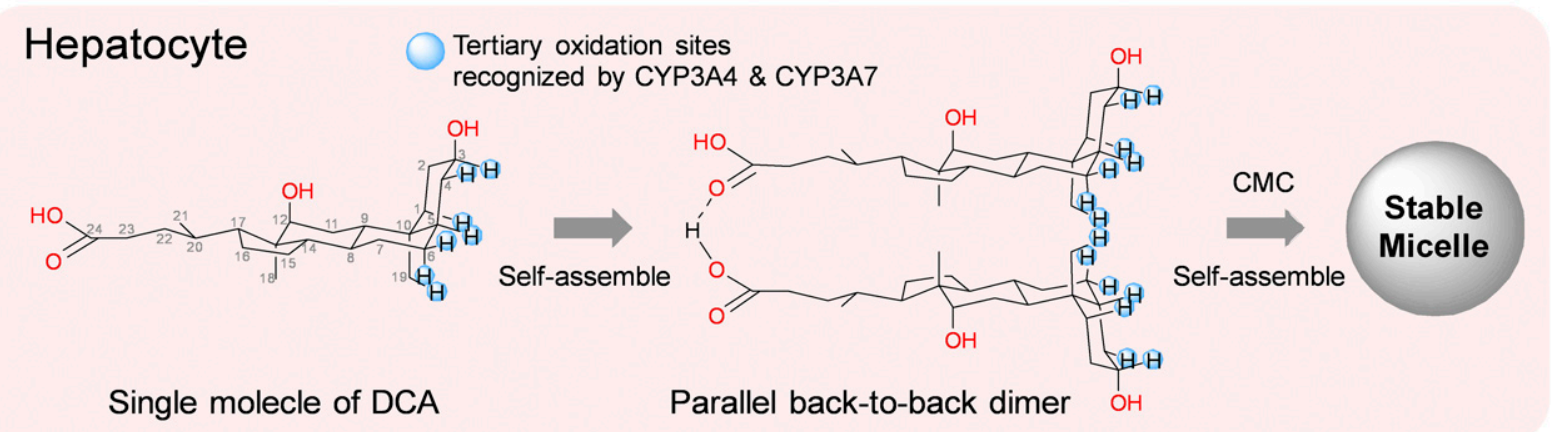

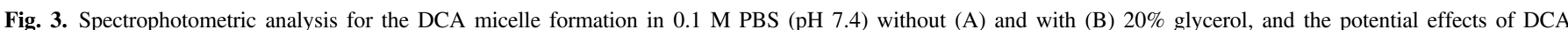

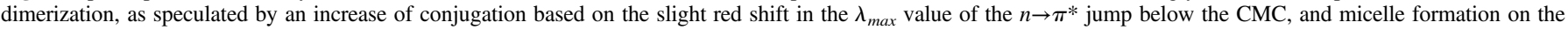
CYP3A-mediated oxidation in hepatocytes (C). the corrected data points were negative due to the system and analytical errors. Even so, the correlation analysis has provided a proof-of-concept evidence that DCA 19-hydroxylation is an in vitro marker reaction for CYP3A7 activity, whereas DCA oxidation at other sites can be used as mixed in vitro indicators for CYP3A4 and CYP3A7 activities.

\section{Discussion}

In combination with our recent findings (Zhang et al., 2019), this study confirmed that DCA is a selective substrate of both CYP3A4 and CYP3A7. Although the CYP3A-catalyzed DCA oxidation is not as robust as that for DHEA (Stevens et al., 2003), testosterone (Kandel et al., 2017), and other drug substrates (Williams et al., 2002), DCA has been furnished as a new probe substrate of CYP3A without contributions from CYP3A5 and other known P450 enzymes. Interestingly, CYP3A4 and CYP3A7 exhibited subtle differences in stereoselectivity and kinetics for DCA oxidation. A positive cooperativity of CYP3A4 and a lack of cooperativity of CYP3A7 were demonstrated by either activity assay or binding assay. CYP3A7 favors the oxidation at C-19, C- $4 \beta, \mathrm{C}-6 \alpha, \mathrm{C}-3 \beta$, and $\mathrm{C}-1 \beta$, whereas $\mathrm{CYP} 3 \mathrm{~A} 4$ favors the oxidation at $\mathrm{C}-5 \beta$ and $\mathrm{C}-6 \beta$. The
B
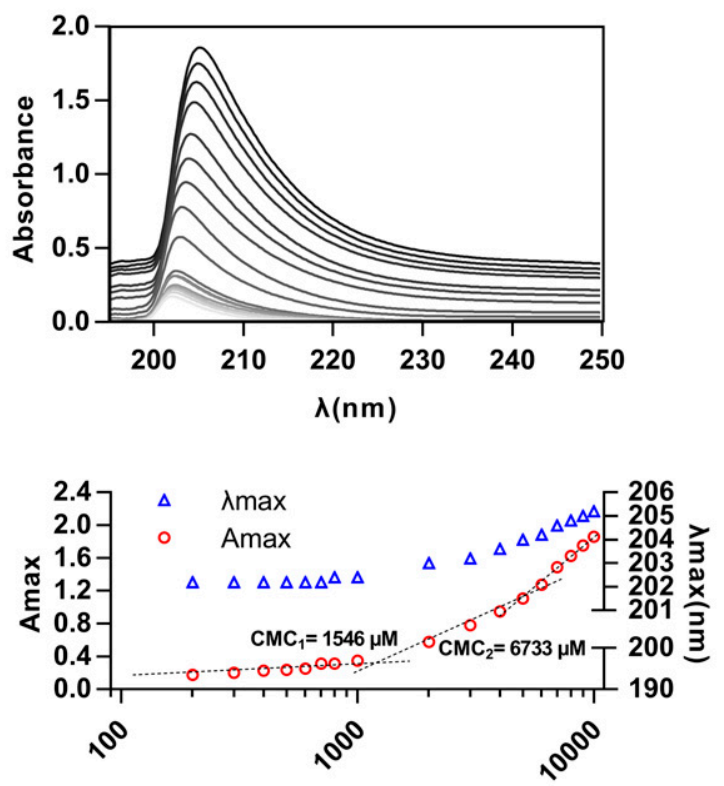

$[\mathrm{DCA}](\mu \mathrm{M})$ correlation analysis between testosterone $6 \beta$-hydroxylation and DCA oxidation in a panel of single-donor HLMs. Although the recombinant CYP3A7 shows much lower in vitro clearance for DCA 19-hydroxylation $(3.64 \mathrm{ml} / \mathrm{min}$ per picomole) than DHEA $16 \alpha$-hydroxylation (1317 $\mathrm{ml} / \mathrm{min}$ per picomole) (Stevens et al., 2003 ) and testosterone $2 \alpha$-hydroxylation ( $77 \mathrm{ml} / \mathrm{min}$ per picomole) (Kandel et al., 2017), DCA 19-hydroxylation has an overwhelmingly greater CYP3A7 selectivity than the latter two reactions. Because glycodeoxycholate (GDCA) and taurodeoxycholate (TDCA) have similar stereoselective oxidation pathways and increased metabolite formations compared with those of DCA (Zhang et al., 2019), 19-hydroxylation of GDCA and TDCA is also anticipated to be an in vitro marker reaction of CYP3A7 with improved activity.

Due to the emerging importance of CYP3A7 in pediatric pharmacology (Stevens, 2006), endocrine diseases (Bacsi et al., 2007; Goodarzi et al., 2008), and cancer therapeutics (Johnson et al., 2016), there might be an increasing need for in vivo probes of CYP3A7 activity. 
TABLE 1

Hill kinetic parameters for stereoselective DCA oxidation catalyzed by recombinant CYP3A4, CYP3A5, and CYP3A7 enzymes in bactosomes

\begin{tabular}{|c|c|c|c|c|c|c|c|c|c|}
\hline \multirow{2}{*}{ Parameters } & \multirow{2}{*}{ Enzyme } & \multicolumn{7}{|c|}{ Oxidation Site } & \multirow{2}{*}{ Total } \\
\hline & & $\mathrm{C}-3 \beta$ & $\mathrm{C}-1 \beta$ & $\mathrm{C}-5 \beta$ & C- $6 \alpha$ & C-19 & $\mathrm{C}-4 \beta$ & $\mathrm{C}-6 \beta$ & \\
\hline \multirow{3}{*}{$V_{\max }(\mathrm{pmol} / \mathrm{min}$ per nanomol protein) } & CYP3A4 & 3920 & 1934 & 566 & 115 & ND & 13.12 & 34.35 & \\
\hline & CYP3A5 & 835 & 54.54 & 26.66 & ND & ND & ND & ND & \\
\hline & CYP3A7 & 11,636 & 6071 & 595 & 728 & 987 & 442 & 24.91 & \\
\hline \multirow{3}{*}{$\mathrm{S}_{50}(\mu \mathrm{M})$} & CYP3A4 & 133 & 101 & 102 & 151 & NA & 98.8 & 144 & \\
\hline & CYP3A5 & 406 & 636 & 586 & NA & NA & NA & NA & \\
\hline & CYP3A7 & 216 & 182 & 170 & 361 & 271.3 & 529 & 165.9 & \\
\hline \multirow[t]{3}{*}{$C L_{\text {int }}(\mathrm{ml} / \mathrm{min}$ per picomole protein $)$} & CYP3A4 & 29.41 & 19.22 & 5.56 & 0.76 & NA & 0.13 & 0.24 & 55.33 \\
\hline & CYP3A5 & 2.06 & 0.09 & 0.05 & NA & NA & NA & NA & 2.19 \\
\hline & CYP3A7 & 53.90 & 33.43 & 3.50 & 2.01 & 3.64 & 0.84 & 0.15 & 97.46 \\
\hline \multirow[t]{3}{*}{ Hill coefficient } & CYP3A4 & 1.08 & 1.16 & 1.17 & 1.18 & NA & 1.68 & 1.16 & \\
\hline & CYP3A5 & 0.79 & 0.85 & 0.82 & NA & NA & NA & NA & \\
\hline & CYP3A7 & 0.98 & 0.92 & 0.90 & 0.87 & 0.86 & 0.79 & 1.74 & \\
\hline \multirow[t]{3}{*}{ Goodness of fit $\left(R^{2}\right)$} & CYP3A4 & 0.9994 & 0.9987 & 0.9984 & 0.9962 & NA & 0.9649 & 0.9931 & \\
\hline & CYP3A5 & 0.9974 & 0.9875 & 0.9843 & NA & NA & NA & NA & \\
\hline & CYP3A7 & 0.9995 & 0.9989 & 0.9977 & 0.9981 & 0.9989 & 0.9971 & 0.9746 & \\
\hline
\end{tabular}

NA, not applicable; ND, not detected.

DCA is endogenous to the host-gut microbial symbiote, showing promise to become a noninvasive probe that is superior to foreign probes. On the other hand, similar to the foreign probe drugs, DCA also shows theoretical advantage over the other host-synthesized steroid substrates, such as cortisol, because it is continuously produced by gut bacteria and is "exogenous" to the host genome. Our recent work preliminarily showed that the frequency of individuals with higher urinary DCA- $4 \beta$-ol/DCA ratio in a small adult population $(n=58)$ was consistent with the CYP3A7expressing frequency $(10 \%)$ in a population including 59 individuals
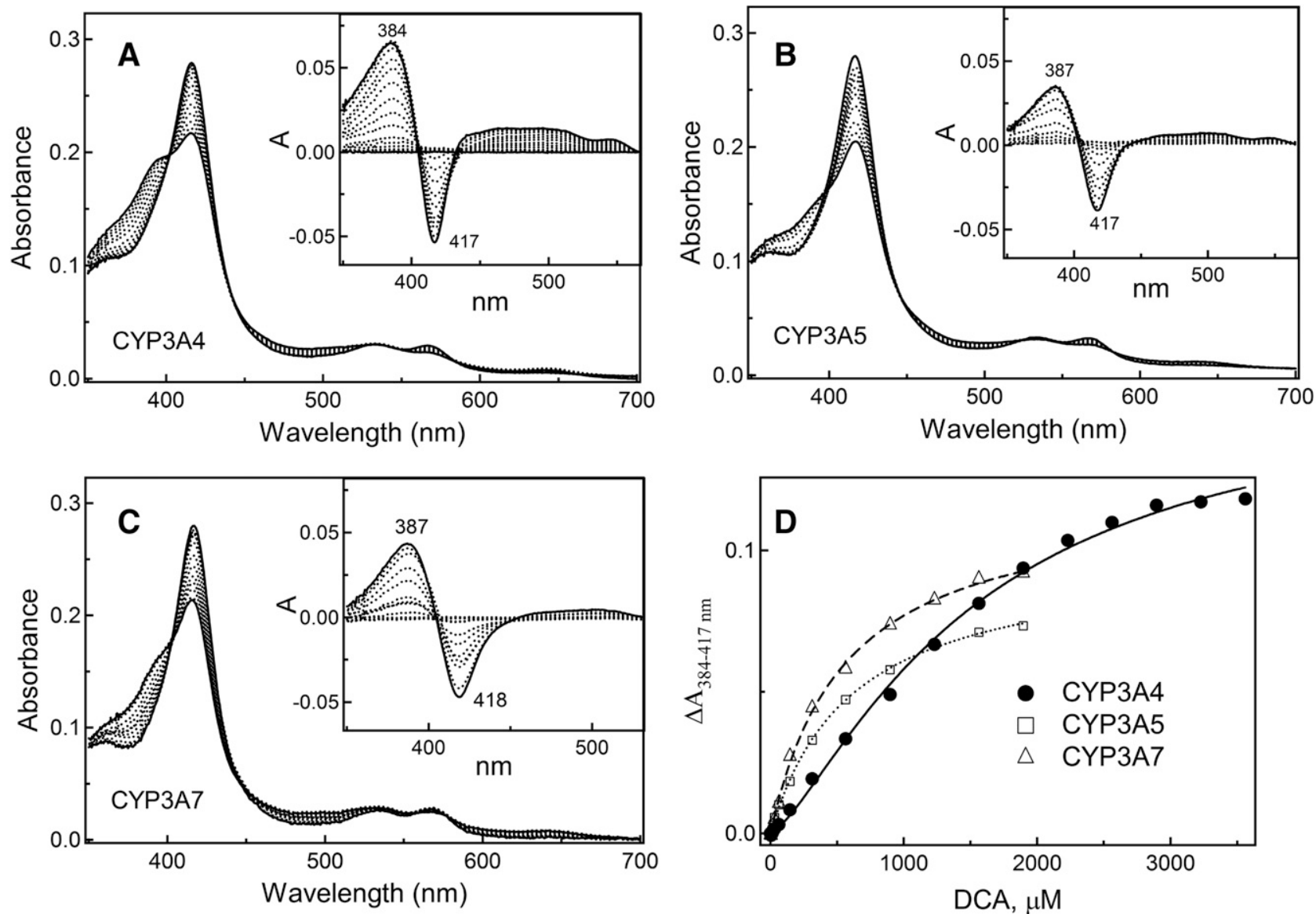

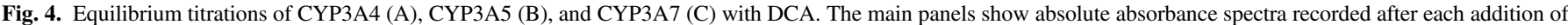

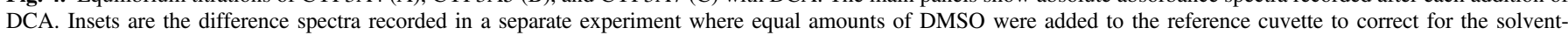

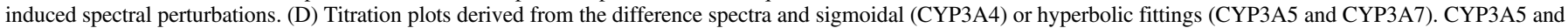
CYP3A7 were less stable than CYP3A4 and were precipitated at $>2000 \mu \mathrm{M}$ DCA. 
TABLE 2

Parameters for DCA binding to isolated recombinant CYP3A enzymes

\begin{tabular}{ccc}
\hline Enzyme & Hill Coefficient & $\mathrm{S}_{50}(\mu \mathrm{M})$ \\
\hline CYP3A4 & $1.45 \pm 0.11$ & $1540 \pm 150$ \\
CYP3A5 & $0.99 \pm 0.01$ & $560 \pm 80$ \\
CYP3A7 & $0.98 \pm 0.03$ & $510 \pm 10$ \\
\hline
\end{tabular}

Values represent an average of three measurements \pm S.E.M.

(Sim et al., 2005). However, DCA-19-ol, which was produced simultaneously but at much higher levels than DCA- $4 \beta$-ol in single-donor HLMs (Fig. 5), was detected only at a trace level in serum and urine samples from the tested population (Zhang et al., 2019). The in vitro-in vivo inconsistency of DCA 19-hydroxylation is believed to be likely associated with the subsequent conversion of DCA-19-ol into another unknown metabolite. Comparative studies of BA metabolome in the CYP3A7-expressing population (newborn and infants) will be useful to understand why DCA-19-ol disappears in the BA pool of adults but DCA- $4 \beta$-ol does not.

DCA is an amphipathic, detergent-like molecule that has been known for decades as a denaturation agent that converts the active P450 to inactive P420 (Gillette et al., 1957; Omura and Sato, 1964a,b). In this work, the deactivation of CYP3A was consistently observed in the activity and binding assays when DCA levels were near or higher than its CMC (about $1500 \mu \mathrm{M}$ ). Below the CMC, DCA oxidation by the recombinant CYP3A4 gradually decreased at 300-1000 $\mu \mathrm{M}$, whereas the activities of recombinant CYP3A7 were abnormally elevated at 500 and $750 \mu \mathrm{M}$, as seen from the extrapolated Hill curves in Fig. 2. Interestingly, we also observed a slight red shift in the $\lambda$ max of $n \rightarrow \pi^{*}$ jump below the CMC (Fig. $3 \mathrm{~A}$ ), indicating an increase of conjugation by establishing the intermolecular hydrogen bonds between DCA molecules. This phenomenon was consistent with the findings of a previous report on the formation of small aggregates, such as dimers, of TDCA (Funasaki et al., 1994). Therefore, the abnormally elevated oxidation activities of CYP3A7 enzymes measured at intermediate DCA concentrations (500 and $750 \mu \mathrm{M}$ ) might be associated, at least in part, with an altered affinity for the DCA multimers. Due to small size and susceptibility to external influences, the absolute structural configuration of bile salt multimer remains in debate (Funasaki et al., 2004, 2005; Galantini et al., 2005). In our opinion, the parallel back-to-back structure involving intermolecular hydrogen bonds and intermolecular hydrophobic interactions may provide a better explanation for the regioselective oxidation sites of DCA(Fig. 3C).

In conclusion, this study reported both the stereoselective oxidation kinetics of DCA in recombinant and microsomal CYP3A enzymes and the binding parameters of DCA with isolated CYP3A enzymes. It was confirmed that DCA 19-hydroxylation is an in vitro marker of CYP3A7 activity, whereas DCA oxidation at other sites shows mixed indicators for CYP3A4 and CYP3A7 activity. Additional investigations are required to study the in vitro CYP3A marker efficacies of the $N$-acylamidated forms of DCA (GDCA and TDCA), the in vitro-in vivo inconsistency of DCA 19-hydroxylation, and the interaction of CYP3A isoforms with DCA species during monomer-multimer-micelle transition. In summary, we believe that deeper understanding of the kinetic and functional roles of CYP3A in disposing the gut bacteriaderived secondary BAs may help to decode the structural and functional mechanisms of these important drug-metabolizing enzymes.
A

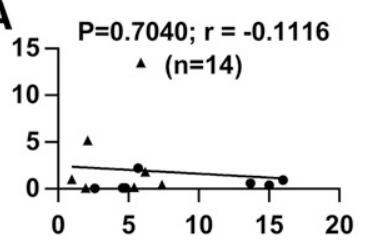

- Female
$\Delta$ Male

B
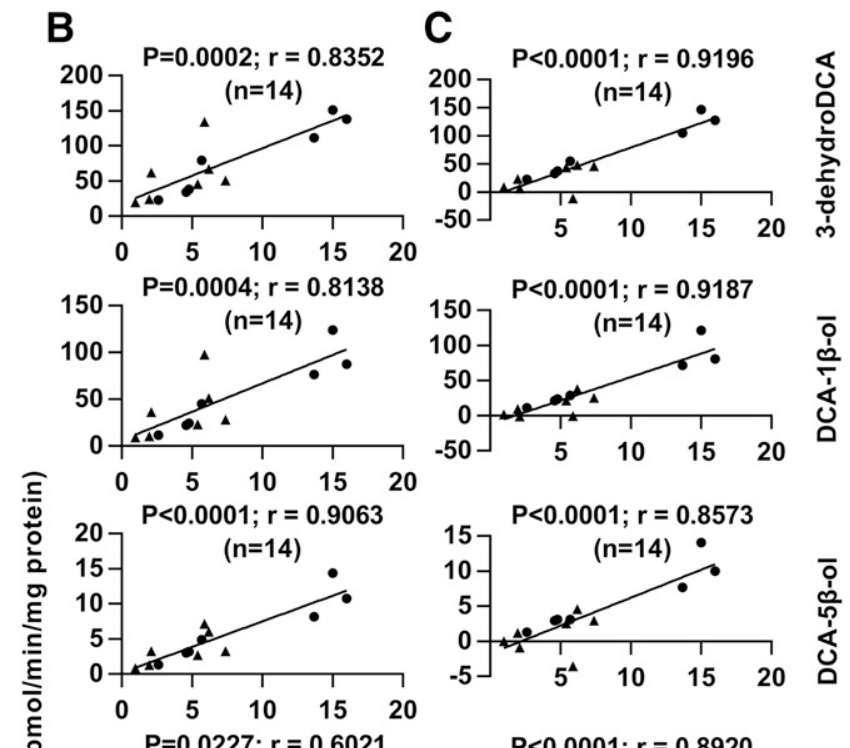

는 10
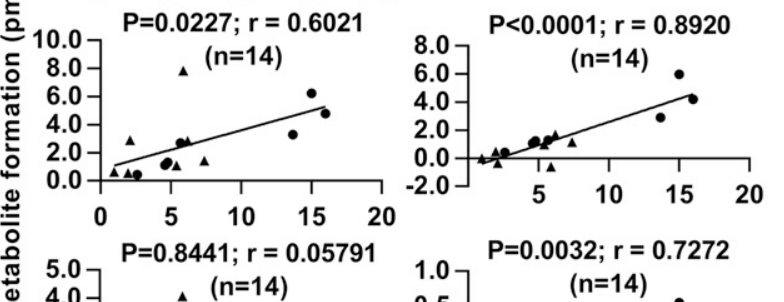

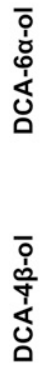
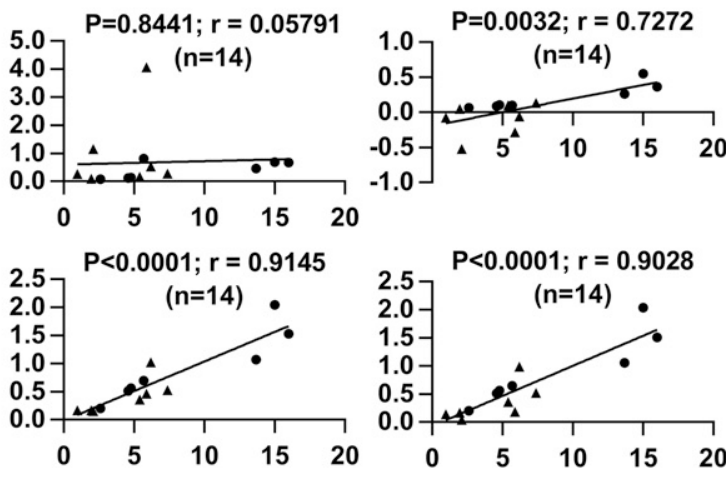

$6 \beta$-hydroxytestosterone formation ( $\mathrm{nmol} / \mathrm{min} / \mathrm{mg}$ protein)

Fig. 5. Correlation of testosterone $6 \beta$-hydroxylation with DCA 19-hydroxylation (A) and DCA oxidation at other sites in 14 single-adult donor HLMs before (B) and after (C) correction by subtracting the CYP3A7 contributions, which was achieved by calculating the CYP3A7 protein levels in single-donor HLMs and estimating the respective activities derived from CYP3A7 according to the activity data acquired in the recombinant CYP3A7 enzymes at $50 \mu \mathrm{M}$ DCA.

\section{Acknowledgments}

K.L. thanks Fan Chen for companionship and support over the past decades.

\section{Authorship Contributions}

Participated in research design: Sevrioukova and Lan.

Conducted experiments: Chen, Zhang, Zhu, Tan, Lin, Wang, Yin, Gao, Su, $\mathrm{Xu}$, and Sevrioukova.

Performed data analysis: Zhang, Sevrioukova, and Lan. 
Wrote or contributed to the writing of the manuscript: Chen, Zhang, Liu, Jia, Sevrioukova, and Lan.

\section{References}

Bácsi K, Kósa JP, Borgulya G, Balla B, Lazáry A, Nagy Z, Horváth C, Speer G, and Lakatos P (2007) CYP3A7*1C polymorphism, serum dehydroepiandrosterone sulfate level, and bone mineral density in postmenopausal women. Calcif Tissue Int $\mathbf{8 0}$ 154-159.

Dawson PA and Karpen SJ (2015) Intestinal transport and metabolism of bile acids. J Lipid Res 56 1085-1099.

de Montellano PRO (2009) Cytochrome P-450: Structure, Mechanism, and Biochemistry, Springer, Berlin.

Drozdzik M, Busch D, Lapczuk J, Müller J, Ostrowski M, Kurzawski M, and Oswald S (2018) Protein abundance of clinically relevant drug-metabolizing enzymes in the human liver and intestine: a comparative analysis in paired tissue specimens. Clin Pharmaco Ther 104:515-524.

Funasaki N, Fukuba M, Kitagawa T, Nomura M, Ishikawa S, Hirota S, and Neya S (2004) Twodimensional NMR study on the structures of micelles of sodium taurocholate. J Phys Chem B 108:438-443.

Funasaki N, Fukuba M, Kitagawa T, Nomura M, Ishikawa S, Hirota S, and Neya S (2005) Reply to the comment on "Two-dimensional NMR study on the structures of micelles of sodium taurocholate". J Phys Chem B 109:9851-9852.

Funasaki N, Ueshiba R, Hada S, and Neya S (1994) Stepwise self-association of sodium taurocholate and taurodeoxycholate as revealed by chromatography. J Phys Chem $\mathbf{9 8}$ $11541-11548$

Galantini L, Giglio E, and Pavel NV (2005) Comment on "Two-dimensional NMR study on the structures of micelles of sodium taurocholate". J Phys Chem B 109:9849-9850.

Gellner K, Eiselt R, Hustert E, Arnold H, Koch I, Haberl M, Deglmann CJ, Burk O, Buntefuss D, Escher S, et al. (2001) Genomic organization of the human CYP3A locus: identification of a new, inducible CYP3A gene. Pharmacogenetics 11:111-121.

Gillette JR, Brodie BB, and La Du BN (1957) The oxidation of drugs by liver microsomes: on the role of TPNH and oxygen. J Pharmacol Exp Ther 119:532-540.

Goodarzi MO, Xu N, and Azziz R (2008) Association of CYP3A7*1C and serum dehydroepiandrosterone sulfate levels in women with polycystic ovary syndrome. J Clin Endocrinol Metab 93:2909-2912.

Hsu MH, Savas U, and Johnson EF (2018) The X-ray crystal structure of the human monooxygenase cytochrome P450 3A5-ritonavir complex reveals active site differences between P450s 3A4 and 3A5. Mol Pharmacol 93:14-24.

Johnson N, De Ieso P, Migliorini G, Orr N, Broderick P, Catovsky D, Matakidou A, Eisen T, Goldsmith C, Dudbridge F, et al. (2016) Cytochrome P450 allele CYP3A $7 * 1 \mathrm{C}$ associates with adverse outcomes in chronic lymphocytic leukemia, breast, and lung cancer. Cancer Res 76: $1485-1493$.

Kandel SE, Han LW, Mao Q, and Lampe JN (2017) Digging deeper into CYP3A testosterone metabolism: kinetic, regioselectivity, and stereoselectivity differences between CYP3A4/5 and CYP3A7. Drug Metab Dispos 45:1266-1275.

Kitada M, Kamataki T, Itahashi K, Rikihisa T, and Kanakubo Y (1987a) P-450 HFLa, a form of cytochrome P-450 purified from human fetal livers, is the 16 alpha-hydroxylase of dehydroepiandrosterone 3-sulfate. J Biol Chem 262:13534-13537.

Kitada M, Kamataki T, Itahashi K, Rikihisa T, and Kanakubo Y (1987b) Significance of cytochrome P-450 (P-450 HFLa) of human fetal livers in the steroid and drug oxidations. Biochem Pharmacol 36:453-456.

Koch I, Weil R, Wolbold R, Brockmöller J, Hustert E, Burk O, Nuessler A, Neuhaus P, Eichelbaum $M$, Zanger U, et al. (2002) Interindividual variability and tissue-specificity in the expression of cytochrome P450 3A mRNA. Drug Metab Dispos 30:1108-1114.

Lacroix D, Sonnier M, Moncion A, Cheron G, and Cresteil T (1997) Expression of CYP3A in the human liver--evidence that the shift between CYP3A7 and CYP3A4 occurs immediately after birth. Eur J Biochem 247:625-634.

Leeder JS, Gaedigk R, Marcucci KA, Gaedigk A, Vyhlidal CA, Schindel BP, and Pearce RE (2005) Variability of CYP3A7 expression in human fetal liver. J Pharmacol Exp Ther 314: 626-635.

Liu YT, Hao HP, Liu CX, Wang GJ, and Xie HG (2007) Drugs as CYP3A probes, inducers, and inhibitors. Drug Metab Rev 39:699-721.
Michaels S and Wang MZ (2014) The revised human liver cytochrome P450 "Pie": absolute protein quantification of CYP4F and CYP3A enzymes using targeted quantitative proteomics. Drug Metab Dispos 42:1241-1251.

Niwa T, Murayama N, Imagawa Y, and Yamazaki H (2015) Regioselective hydroxylation of steroid hormones by human cytochromes P450. Drug Metab Rev 47:89-110.

Omura T and Sato R (1964a) The carbon monoxide-binding pigment of liver microsomes. I. Evidence for its hemoprotein nature. J Biol Chem 239:2370-2378.

Omura T and Sato R (1964b) The carbon monoxide-binding pigment of liver microsomes. II. Solubilization, purification, and properties. J Biol Chem 239:2379-2385.

Paine MF, Hart HL, Ludington SS, Haining RL, Rettie AE, and Zeldin DC (2006) The human intestinal cytochrome P450 "pie". Drug Metab Dispos 34:880-886.

Patki KC, Von Moltke LL, and Greenblatt DJ (2003) In vitro metabolism of midazolam, triazolam, nifedipine, and testosterone by human liver microsomes and recombinant cytochromes p450: role of cyp3a4 and cyp3a5. Drug Metab Dispos 31:938-944.

Reis S, Moutinho CG, Matos C, de Castro B, Gameiro P, and Lima JL (2004) Noninvasive methods to determine the critical micelle concentration of some bile acid salts. Anal Biochem 334:117-126.

Rendic S and Guengerich FP (2015) Survey of human oxidoreductases and cytochrome P450 enzymes involved in the metabolism of xenobiotic and natural chemicals. Chem Res Toxicol $\mathbf{2 8}$ : $38-42$.

Ridlon JM, Kang DJ, and Hylemon PB (2006) Bile salt biotransformations by human intestinal bacteria. J Lipid Res 47:241-259.

Russell DW (2003) The enzymes, regulation, and genetics of bile acid synthesis. Annu Rev Biochem 72:137-174

Schuetz JD, Kauma S, and Guzelian PS (1993) Identification of the fetal liver cytochrome CYP3A7 in human endometrium and placenta. J Clin Invest 92:1018-1024.

Sevrioukova IF (2017) High-level production and properties of the cysteine-depleted cytochrome P450 3A4. Biochemistry 56:3058-3067.

Shimada T, Yamazaki H, Mimura M, Inui Y, and Guengerich FP (1994) Interindividual variations in human liver cytochrome P-450 enzymes involved in the oxidation of drugs, carcinogens and toxic chemicals: studies with liver microsomes of 30 Japanese and 30 Caucasians. J Pharmacol Exp Ther 270:414-423.

Sim SC, Edwards RJ, Boobis AR, and Ingelman-Sundberg M (2005) CYP3A7 protein expression is high in a fraction of adult human livers and partially associated with the CYP3A7*1C allele. Pharmacogenet Genomics 15:625-631.

Stevens JC (2006) New perspectives on the impact of cytochrome P450 3A expression for pediatric pharmacology. Drug Discov Today 11:440-445.

Stevens JC, Hines RN, Gu C, Koukouritaki SB, Manro JR, Tandler PJ, and Zaya MJ (2003) Developmental expression of the major human hepatic CYP3A enzymes. J Pharmacol Exp Ther 307:573-582.

Wienkers LC and Heath TG (2005) Predicting in vivo drug interactions from in vitro drug discovery data. Nat Rev Drug Discov 4:825-833.

Williams JA, Ring BJ, Cantrell VE, Jones DR, Eckstein J, Ruterbories K, Hamman MA, Hall SD, and Wrighton SA (2002) Comparative metabolic capabilities of CYP3A4, CYP3A5, and CYP3A7. Drug Metab Dispos 30:883-891.

Yin S, Su M, Xie G, Li X, Wei R, Liu C, Lan K, and Jia W (2017) Factors affecting separation and detection of bile acids by liquid chromatography coupled with mass spectrometry in negative mode. Anal Bioanal Chem 409:5533-5545.

Zhang J, Gao LZ, Chen YJ, Zhu PP, Yin SS, Su MM, Ni Y, Miao J, Wu WL, Chen H, et al. (2019) Continuum of host-gut microbial co-metabolism: host CYP3A4/3A7 are responsible for tertiary oxidations of deoxycholate species. Drug Metab Dispos 47:283-294.

Zhu P, Zhang J, Chen Y, Yin S, Su M, Xie G, Brouwer KLR, Liu C, Lan K, and Jia W (2018) Analysis of human $\mathrm{C} 24$ bile acids metabolome in serum and urine based on enzyme digestion of conjugated bile acids and LC-MS determination of unconjugated bile acids. Anal Bioanal Chem 410:5287-5300.

Address correspondence to: Dr. Irina F. Sevrioukova, Department of Molecular Biology and Biochemistry, University of California, Irvine, Irvine, CA 92697-3900. E-mail: sevrioui@uci.edu; or Dr. Ke Lan, West China School of Pharmacy, Sichuan University, No.17 People's South Road, Chengdu 610041, People's Republic of China. E-mail: lanwoco@scu.edu.cn 